\title{
Atomic Ordering in Self-assembled Epitaxial II-VI and IV-VI Compound Semiconductor Quantum Dot Systems
}

\author{
Peter Möck ${ }^{1}$, Klaus Pierz ${ }^{2}$, Teya Topuria ${ }^{3}$, Nigel D. Browning ${ }^{3}$, Huizhen $\mathrm{Wu}^{4 *}$, and Patrick J. McCann ${ }^{4}$ \\ ${ }^{1}$ Portland State University, Department of Physics, P.O. Box 751, Portland, OR 97207-0751, pmoeck@ pdx.edu \\ ${ }^{2}$ Physikalisch-Technische Bundesanstalt Braunschweig, Bundesallee 100, 38116 Braunschweig, Federal \\ Republic of Germany \\ ${ }^{3}$ University of Illinois at Chicago, Department of Physics, 845 W. Taylor Street, Chicago, Illinois 60607-7059 \\ ${ }^{4}$ University of Oklahoma, School of Electrical and Computer Engineering, Norman, OK 73019, * now at \\ Department of Physics, Zhejiang University, \#34 Tian Mu Shan Road, Hangzhou, Zhejiang 310028, People's \\ Republic of China
}

\begin{abstract}
Transmission electron microcopy (TEM) in both the parallel illumination and scanning probe mode revealed atomically ordered entities within a 5 to $250 \mathrm{~nm}$ range in a IV-VI and a II-VI compound semiconductor quantum dot (QD) system. While the II-VI system was a nominal [001] CdSe/( $\left.\mathrm{Mn}_{0.1} \mathrm{Zn}_{0.9}\right) \mathrm{Se}$ multilayer structure with the QDs embedded, the IV-VI system nominally consisted of [111] PbSe islands. The comparison of photoluminescence (PL) spectra from the CdSe/( $\left(\mathrm{Mn}_{0.1} \mathrm{Zn}_{0.9}\right) \mathrm{Se}$ structure with those of a reference structure, that was grown to the same nominal specification under otherwise identical conditions except that no Mn was incorporated into the cladding layers, revealed for the former sample two peaks at approximately 2 and $2.1 \mathrm{eV}$. We tentatively attribute these two PL peaks to two groups of atomically ordered entities.
\end{abstract}

\section{INTRODUCTION}

Since 1985 it has been well known that atomic ordering and phase separation exists in basically all heteroepitaxial III-V compound semiconductor structures [1]. In recent years, atomic ordering and phase separation has also been observed in several heteroepitaxial II-VI systems, e.g. (Cd,Zn)Se [2-4], (Cd,Mn,Zn)Se [3,5-7], $(\mathrm{Fe}, \mathrm{Zn}) \mathrm{Se}$ [8], $(\mathrm{Cd}, \mathrm{Zn}) \mathrm{Te}$ [9-11], and $\mathrm{Cd}(\mathrm{Se}, \mathrm{Te})$ [12]. Atomic ordering and phase separation in IV-VI systems has, to the best of our knowledge, only been reported once in epitaxial $(\mathrm{Pb}, \mathrm{Eu}) \mathrm{Te}$ [13].

There is the tacit assumption throughout the QD community that the structure of epitaxial self assembled semiconductor QDs is always determined by the structural prototype of the substrate. While most III-V and II-VI QDs are supposed to exist in the sphalerite structure, $\mathrm{Pb}(\mathrm{Se}, \mathrm{Te}) \mathrm{QDs}$ are supposed to exist in the halite structure. Since the operation of the Stranski-Krastanow growth mode (and its variants) has been shown to be based on alloying [14,15], one has to conclude that self-assembled semiconductor QDs which grow in these mode(s) are always alloyed. (Note also that this conclusion it well justified by experimental studies on a variety of element and compound semiconductor QD systems). In line with the tacit assumption above, the mixed cations or anions in these QD structures are expected to be distributed randomly over their respective sublattices.

Over recent years, there has, however, been an accumulation of experimental observations [3-7,16] that suggest that these assumptions are not always justified. In this paper we report on high resolution phase contrast TEM (HRTEM), selected area high energy transmission electron diffraction (SAED), and atomic resolution Z-contrast scanning TEM (Z-STEM) investigations on a nominal CdSe/( $\left.\mathrm{Mn}_{0.1} \mathrm{Zn}_{0.9}\right) \mathrm{Se}$ multilayer QD structure and a nominal $\mathrm{PbSe}$ island (i.e. QD predecessor) on PbTe structure. Complementary PL studies from the $\mathrm{CdSe} /\left(\mathrm{Mn}_{0.1} \mathrm{Zn}_{0.9}\right) \mathrm{Se}$ structure and a reference structure, that was grown to the same nominal specification under otherwise identical conditions except that no $\mathrm{Mn}$ was incorporated into the cladding layers, are also reported.

\section{EXPERIMENTAL DETAILS}

The two II-VI QD structures of this study were grown at the University of Notre Dame by means of molecular beam epitaxy (MBE) in a Riber 32 R\&D machine equipped with elemental sources. For both samples, nominally $82.5 \mathrm{~nm}$ of $\mathrm{ZnSe}$ were first deposited homoepitaxially onto the $\mathrm{ZnSe} / \mathrm{GaAs}$ pseudo-substrate at $300{ }^{\circ} \mathrm{C}$. A multilayer structure of eight sequences of 10 monolayers (ML), i.e. $2.8 \mathrm{~nm}$, of (Mn, Zn)Se cladding layer with either $10 \% \mathrm{Mn}$ (sample A) or $0 \% \mathrm{Mn}$ (sample B) and $0.3 \mathrm{ML}(0.09 \mathrm{~nm}) \mathrm{CdSe}$ sheet (both samples) was then deposited at $350^{\circ} \mathrm{C}$. While the cladding layers for both samples were grown at a deposition rate of $1 \mathrm{ML} \mathrm{s}^{-1}$, the CdSe sheets were grown 
at a deposition rate of $0.038 \mathrm{ML} \mathrm{s}^{-1}$. As the final $\left(9^{\text {th }}\right)$ cladding layer of the multilayer structure, $10 \mathrm{ML}(\mathrm{Mn}, \mathrm{Zn}) \mathrm{Se}$ with either $10 \% \mathrm{Mn}$ (sample A) or $0 \% \mathrm{Mn}$ (sample B) were deposited and finally a $\mathrm{ZnSe}$ capping layer of $50 \mathrm{~nm}$ thickness was grown at $350{ }^{\circ} \mathrm{C}$ for both samples. While within the $26.19 \mathrm{~nm}$ thick multilayer structure of sample A, there are, on average, nominally $87.3 \%$ of the cation sites occupied by $\mathrm{Zn}, 9.7 \%$ by $\mathrm{Mn}$ and $3 \%$ by $\mathrm{Cd}$, the otherwise identical multilayer structure of sample B does not contain any Mn.

The nominal PbSe islands on PbTe were grown by means of MBE at the University of Oklahoma using PbTe, $\mathrm{PbSe}$ and Se sources. Freshly cleaved (111) oriented $\mathrm{BaF}_{2}$ was used as the substrate. After desorption of surface layers for 15 minutes at $500{ }^{\circ} \mathrm{C}$, a $2.67 \mu \mathrm{m}$ thick, lattice matched $\mathrm{PbTe}_{0.22} \mathrm{Se}_{0.78}$ buffer layer was grown at $400{ }^{\circ} \mathrm{C}$ and a growth rate of $0.278 \mathrm{~nm} \mathrm{~s}^{-1}$. At the same temperature, $1.8 \mu \mathrm{m}$ Se doped PbTe was grown at a rate of $0.167 \mathrm{~nm} \mathrm{~s}^{-1}$. Then the temperature was lowered to $380^{\circ} \mathrm{C}$ and $0.8 \mathrm{~nm}(2.6 \mathrm{ML}) \mathrm{PbSe}$ was deposited at a rate of $0.027 \mathrm{~nm} \mathrm{~s}^{-1}$. The sample was finally cooled down to room temperature at a rate of $1 \mathrm{~K} \mathrm{~s}^{-1}$. Both kinds of samples have, thus, in common a low growth temperature, which is for MBE grown II-VI and IV-VI compound semiconductors quite common, and a growth rate of below $0.1 \mathrm{ML} \mathrm{s}^{-1}$ for the QD or QD predecessor structures.

From sample A i.e. the nominal CdSe/(Mn,Zn)Se QD structure, [001] plan-view and <110〉 cross-section TEM/STEM specimens were prepared for electron microscopical investigations by standard mechanical grinding and ion-milling techniques approximately three years after the growth. From sample $\mathrm{C}$, i.e. the $\mathrm{Pb}(\mathrm{Se}, \mathrm{Te}) \mathrm{QDs}$ predecessor structure, [111] plan-view TEM/STEM specimens were prepared by standard mechanical grinding and ion-milling techniques approximately eight months after the growth. The specimens were analyzed in the parallel illumination mode using a JEOL JEM-3010 TEM at $300 \mathrm{kV}$ and in the scanning probe Z-contrast mode at $200 \mathrm{kV}$ utilizing the JEOL JEM-2010F field emission STEM/TEM at the University of Illinois at Chicago [17].

Complementary PL measurements were performed on the II-VI multilayer QD structures at a temperature of $6 \mathrm{~K}$. $\mathrm{Ar}^{+}$laser light utilizing both the $488 \mathrm{~nm}$ line and ultraviolet light of around $350 \mathrm{~nm}$ wavelength was used at varying power densities for the excitation of the spectra. The PL emission light was dispersed in a $1 \mathrm{~m}$ single-grating spectrometer and detected with a cooled (In,Ga)As photomultiplier tube using conventional lock-in techniques. The ZnSe band gap being at $6 \mathrm{~K}$ approximately $2.8 \mathrm{eV}$, excitation with $350 \mathrm{~nm}$ light can be considered as non-resonant above this band gap and is expected to reveal most radiatively active structures in the samples. $488 \mathrm{~nm}$ light excitation, on the other hand, can be considered as resonant excitation below the ZnSe band gap, is typically orders of magnitude weaker than non-resonant excitation over the band gap, and selective of specific structures.

\section{RESULTS AND DISCUSSIONS}

\section{A. II-VI Quantum Dots}

HRTEM, SAED, and Z-STEM studies on sample A revealed the coexistence of large double period $\pm(-110)$, Fig. 1a, and medium sized double period $\pm(-111)$ ordered agglomerates, Fig. 1b, with other types of long range atomic ordering, that resulted in $\pm\left\{0-3 / 2{ }^{3} / 2\right\}, \pm\{?-? 0\}$, and $\pm\left\{\begin{array}{lll}? & -? & 1\end{array}\right\}$ superlattice spots in the diffraction patterns, Fig. $1 \mathrm{c},[3,5-7]$. Some three dimensional (3D) double period $\pm(-111)$ ordered agglomerates, Fig. 1b, were observed to be in the size range for quantum confinement to occur $[3,6]$ and we consider these agglomerates to constitute a novel kind of self-assembled semiconductor QD. These QDs should have an enhanced confinement potential since atomic ordering leads typically to reduced band gaps $[1,18]$.

For some parts of the same sample, HRTEM showed correlated, anti-correlated, and uncorrelated stacking of quasi-two dimensional (2D) agglomerates with lateral dimensions of the order of magnitude $5 \mathrm{~nm}$ and the sphalerite structure, showing pronounced strain fields and constituting a kind of ordinarily strained QD [6]. This particular result is in good agreement with a HRTEM study by other authors [19] for a very similar (Cd,Zn)Se/ZnSe multilayer structure (with ZnSe cladding layers of a nominal thickness of $3 \mathrm{~nm}$ and CdSe sheets of nominally the same thickness as in our study).

As the current electron microscopy investigations and the TEM/STEM specimen preparation were performed about three years after the growth, we speculate that the stacked ordinarily strained QDs represent the remains of the original structure. The observation of all of the above mentioned long range order microstructures is in disagreement with the tacit assumption that self-assembled semiconductor QDs always possess the structural prototype of the substrate. As we are unable to link these and previously observed [3-7] long range order microstructures to other possible causes (such as electron beam damage, sample preparation or imaging artifacts [3]), we tentatively assume that it has resulted from thermodynamically driven atomic rearrangements over time. Simple thermodynamical arguments in favor of this assumption are given in ref. [4]. We also observed short range order in III-V QD systems [4] shortly after the growth (but never long range ordered microstructures [20]) and speculate that such short range order may over sufficiently long times grow into long range order, possibly even at room temperature [20]. 

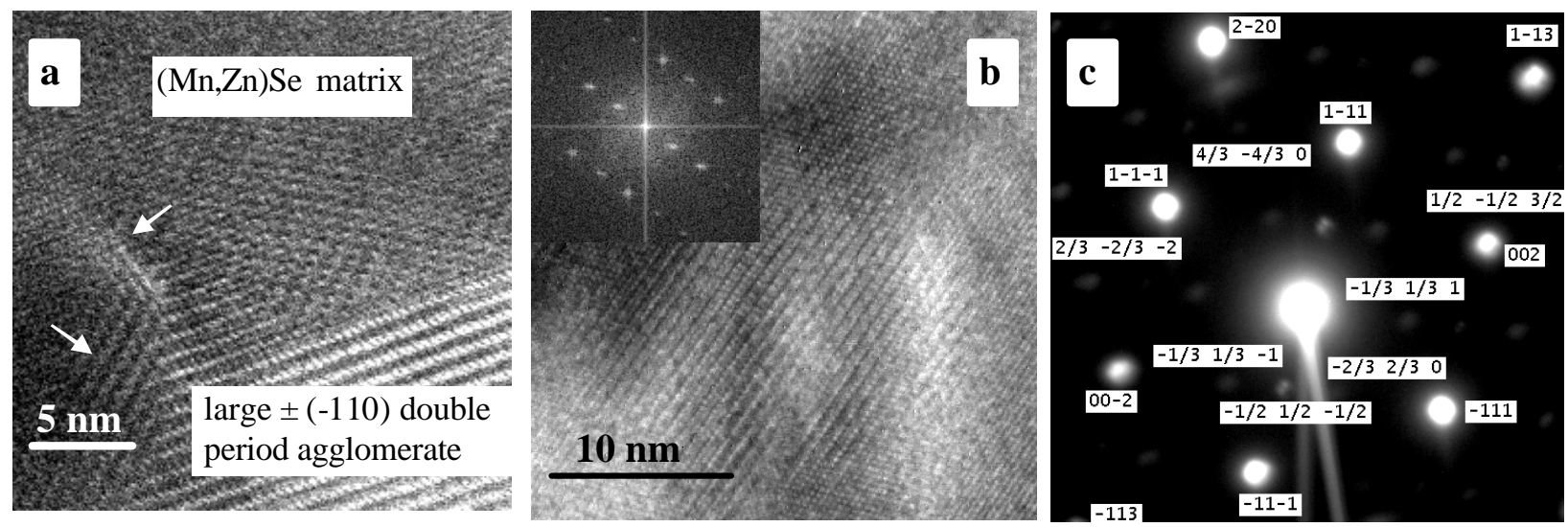

Figure 1. $\langle 110\rangle$ cross section electron micrographs of the nominal $\mathrm{CdSe} /\left(\mathrm{Mn}_{0.1} \mathrm{Zn}_{0.9}\right) \mathrm{Se}$ multilayer structure, sample A; (a) atomic resolution Z-contrast image of differently sized $(\mathrm{Cd}, \mathrm{Mn}, \mathrm{Zn}) \mathrm{Se}$ agglomerates, the matrix and a large double period $\pm(-110)$ agglomerate (that is about $200 \mathrm{~nm}$ in diameter) are labeled, closer inspection of this micrographs shows that there are also two small domains of differently ordered $(\mathrm{Cd}, \mathrm{Mn}, \mathrm{Zn}) \mathrm{Se}$ agglomerates (arrows); (b) HRTEM image of an atomically ordered $50 \% \mathrm{Cd}$ containing QD in (Mn,Zn)Se matrix with inset Fourier transform power spectrum that demonstrates the double period $\pm(-111)$ ordering; (c) SAED pattern showing superlattice reflections. The bright lines in (c) that seem to originate from the transmitted beam are artifacts of the CCD camera used for the recording of this image.
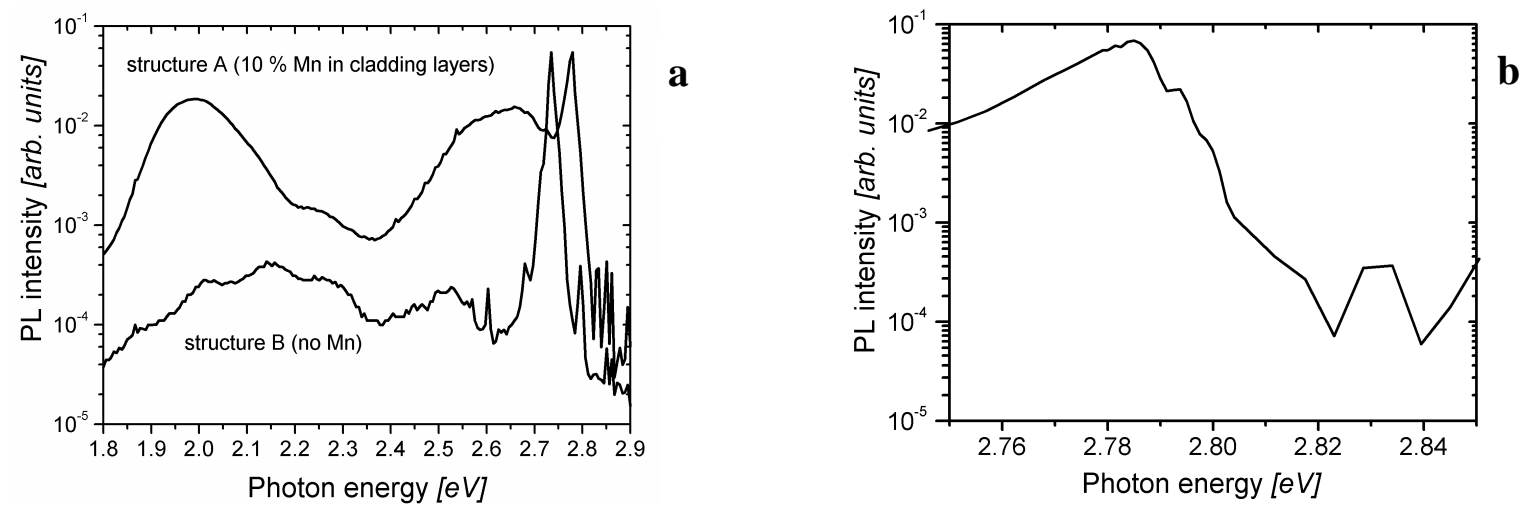

Figure 2. Non-resonant photoluminescence spectra of the II-VI multilayer structures; (a) both samples; (b) higher resolution photoluminescence spectrum of sample A.
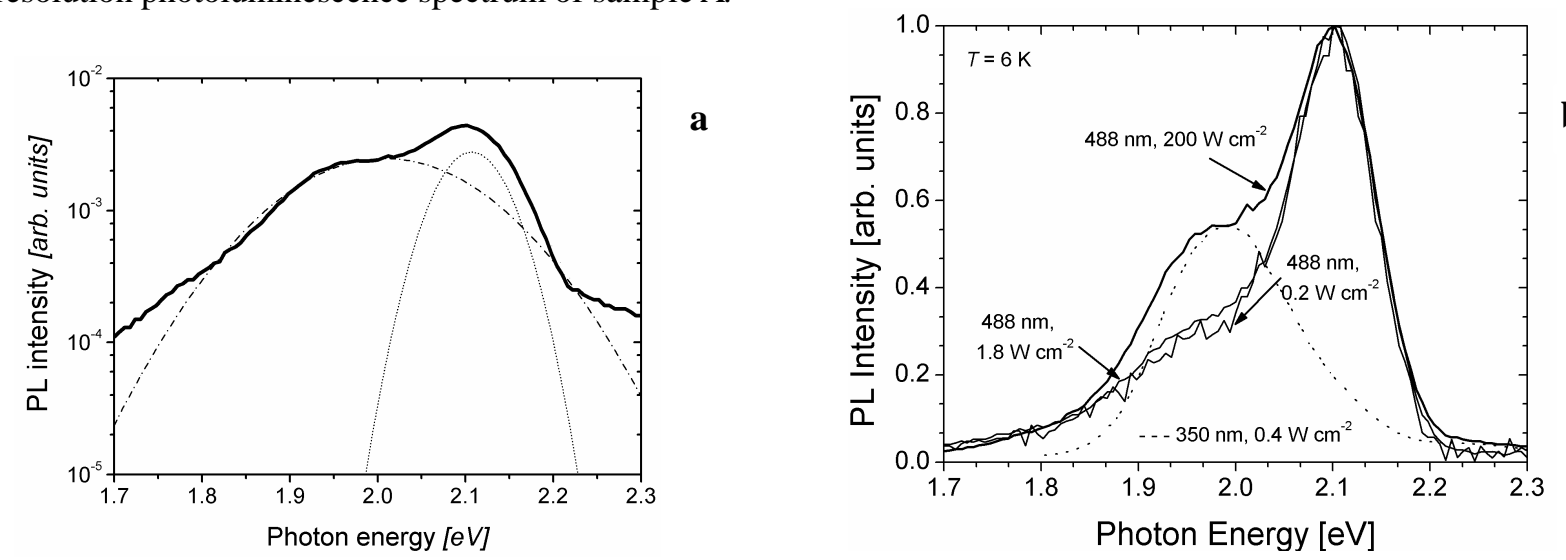

Figure 3. Resonant photoluminescence spectra of sample A; (a) the thin dotted lines represent fits to two Gaussian peaks with a first maximum at $2.107 \mathrm{eV}$ and a corresponding full width at half maximum (FWHM) of $72 \mathrm{meV}$, and a second maximum at $2.0 \mathrm{eV}$ with $202 \mathrm{meV}$ FWHM, (b) varying excitation power densities cause shifts in the relative peak heights of these two peaks (peak heights are normalized, the non-resonant PL spectrum of the same sample in the same energy region is added as a dotted line). 
Having identified large double period $\pm(-110)$ ordered $(\mathrm{Cd}, \mathrm{Mn}, \mathrm{Zn}) \mathrm{Se}$ agglomerates as the by far dominant kind of structural entity per unit volume of the analyzed TEM specimens, it is now interesting to analyze the PL spectra of this sample and compare it with that of the (no Mn containing) reference sample B.

We tentatively suggest that the large and rather broad PL peak at $2 \mathrm{eV}$ in Fig. 2a, that arose when a sample was excited with laser light of approximately $350 \mathrm{~nm}$, is due to the large double period $\pm(-110)$ agglomerates, labeled in Fig. 1a. The $2.1 \mathrm{eV}$ PL peak, Fig. 3a,b, which arose in addition to the $2 \mathrm{eV}$ peak, when a sample was excited with laser light of $488 \mathrm{~nm}$ may be due to the double period $\pm(-111)$ atomically ordered QDs with $50 \% \mathrm{Cd}$ content and a size of approximately $25 \mathrm{~nm}$, Fig. 1b. Fig. $3 \mathrm{~b}$ shows that it is the $2 \mathrm{eV}$ peak which increases in height more strongly with the illumination power density under resonant excitation conditions. Further PL experiments are currently been undertaken with varying resonant excitation wavelengths and power densities in order to prove or disprove if one (or perhaps both) of these low energy PL peaks arose indeed from zero-dimensional entities. Assuming for now that the above assignments of these two low energy peaks to atomically ordered quantum dots and agglomerates are correct, we have to conclude that these long range order microstructures must be distributed throughout rather large regions of sample A. This is because the PL spectra were collected from areas about 6 orders of magnitude larger than the areas that were investigated by means of TEM.

The high energy PL peaks of sample A in Fig. 2a are assigned as follows: $2.78 \mathrm{eV}$ is due to a superposition of the (essentially) 2D remains of the CdSe submonolayers and the excitonic transition in the ZnSe buffer and capping layers, $2.64 \mathrm{eV}$ is due to a large population of small quasi-2D CdSe rich platelets (with sphalerite structure) that act as a first kind of ordinarily strained QD, $2.25 \mathrm{eV}$ could be due to a small population of medium sized 3D CdSe rich QDs (with sphalerite structure) that act as a second kind of ordinarily strained QD [21,22]. Such a coexistence of small quasi-2D and medium sized 3D ordinarily stained QDs has actually been observed in similar samples of the same material system [6,7]. The higher resolution (1.e. smaller entrance and exit slits of the spectrometer) PL spectrum of the high energy region of sample A, Fig. 2b, shows that the $2.78 \mathrm{eV}$ peak in Fig. 2a is indeed due to a superposition of peaks.

This assignment of the peaks is also applicable to the reference structure, sample B, where no Mn was incorporated into the cladding layers. Here, there is a distinct peak at $2.8 \mathrm{eV}$, arising from the excitonic transition in the $\mathrm{ZnSe}$ buffer and capping layers and another high energy peak at $2.74 \mathrm{eV}$, which may be due to rather homogenous 2D layers that resulted from the deposition of the submonolayer CdSe sheets. As these layers seems to be thicker than those of sample A (because the corresponding PL peak is observed at lower photon energies [21]) and significantly more homogenous (and all lower energy peaks are very low in intensity), we speculate that there may be very low (or possibly zero) populations of QDs of all three kinds and of large atomically ordered $\pm(-110)$ double period $(\mathrm{Cd}, \mathrm{Mn}, \mathrm{Zn}) \mathrm{Se}$ agglomerates. Future TEM/STEM investigation of this sample will clarify this point and be reported elsewhere.

Kim et al. observed on nominally pure (i.e. nominally no $\mathrm{Zn}$ or Mn containing) CdSe QDs in $\mathrm{Mn}_{\mathrm{x}} \mathrm{Zn}_{1-\mathrm{x}} \mathrm{Se}$ matrices with $\mathrm{x}=0.05$ to 0.25 a red shift of the PL peak from approximately 2.21 to $2.06 \mathrm{eV}$ with increasing Mn content [23]. Single QD studies of nominally pure CdSe QDs in $\mathrm{Mn}_{0.25} \mathrm{Zn}_{0.75}$ Se matrix by Bacher et al. showed intense PL peaks at approximately $2.09 \mathrm{eV}$, i.e. below the transition between internal $\mathrm{Mn}^{2+}$ states at $2.15 \mathrm{eV}$ [24]. This is similar to the results by Titova et al. [25] where pronounced PL peaks at approximately $2.1 \mathrm{eV}$ were observed for samples grown by a MBE growth procedure where 0.2 to 0.5 monolayers of MnSe were deposited on $\mathrm{ZnSe}$ buffer layers prior to the deposition of the CdSe layer from which nominally pure CdSe QDs formed after the deposition of a ZnSe capping layer [6,7].

None of the above mentioned studies [23-25] confirmed their PL peak assignments with structural analyses on the same samples. Since we indeed observed (110) superlattice spots in SAED patterns of [001] plan views prepared from the samples presented in ref. [25] (and there is no evidence to the contrary from other investigations), we tentatively suggest here that the PL peak at approximately $2.1 \mathrm{eV}$ Titova et al. [25] reported is due to atomically ordered QDs. As expected for atomically ordered entities [1,18], these peaks are at lower energies than the peaks that have previously been attributed to ordinarily strained QDs (be they either truly 3D or quasi-2D) in the similar $(\mathrm{Cd}, \mathrm{Zn}) \mathrm{Se} / \mathrm{ZnSe}$ materials system [21]. For the lack of contradicting structural analysis results, we speculate that our tentative assignment of the peaks at approximately $2 \mathrm{eV}$ and $2.1 \mathrm{eV}$ to atomically ordered agglomerates and atomically ordered QDs might also be applicable to the related peaks around $2.1 \mathrm{eV}$ that Kim et al. [23] and Bacher at al. [24] observed.

We finally note that a peak of the rather similar energy of $2.04 \mathrm{eV}$ has been obtained by cathodoluminescence from atomically ordered $(\mathrm{Cd}, \mathrm{Zn}) \mathrm{Se}$ alloy layers and has been attributed to agglomerates of the $\mathrm{CdZnSe}_{2}$ compound as identified from X-ray diffraction superlattice reflections [2]. PL peaks around $2.05 \mathrm{eV}$ were also observed in $\mathrm{Zn}(\mathrm{S}, \mathrm{Se}) / \mathrm{CdSe} / \mathrm{Zn}(\mathrm{S}, \mathrm{Se})$ quantum well structures (with either 0 or $6 \% \mathrm{~S}$ ) and assigned to some structurally unidentified defects in the $\mathrm{ZnSe}$ and $\mathrm{Zn}(\mathrm{S}, \mathrm{Se}$ ) layers [26]. Atomic ordering (and possibly phase separation) in 
epitaxial $(\mathrm{Cd}, \mathrm{Mn}, \mathrm{Zn})(\mathrm{S}, \mathrm{Se})$ with small or zero Mn content that manifests itself by luminescence peaks between 2 and $2.1 \mathrm{eV}[2,23-26]$, may, after all, not be a rarely occurring phenomenon.

\section{B. IV-VI islands}

Fig. 4a shows differently sized $\mathrm{Pb}(\mathrm{Se}, \mathrm{Te})$ islands. As the diffraction contrast in this image shows, there are two kinds of islands that are obviously strained and we call them ordinarily strained islands. They may, thus, be considered to constitute predecessors of ordinarily strained QDs.

Adjacent to the areas with ordinarily strained islands, there were specimen regions with islands that showed under the same imaging condition contrast that is probably mainly due to absorption, but not consistent with strain field effects, Fig. 4b. The diffraction pattern to Fig. 4b showed a variety of superlattice reflections, Fig. 4c. From the contrast in Fig. $4 b$ and the superlattice reflections in Fig. $4 c$, we infer that these islands may not be under (significant) lattice mismatch strains because they might consist of atomically ordered $\mathrm{Pb}(\mathrm{Se}, \mathrm{Te})$, i.e. represent $\mathrm{Pb}_{\mathrm{x}} \mathrm{Se}_{\mathrm{y}} \mathrm{Te}_{\mathrm{z}}$ compounds with elastic mismatch energy minimizing orientation relationships and lattices.

The same superlattice reflections were also observed in Fourier transform power spectra of high resolution phase contrast images from the same specimen region, which showed indeed fringes of lattices that were distinctly different from those of the halite structural prototype. The $\langle 112\rangle$ zone axis SAED pattern from the same specimen region also showed superlattice spots and streaks perpendicular to the transmitted electron beam direction. These images will be further analyzed and published elsewhere.

Taking into account that about eight months had elapsed between the growth and the TEM analysis of this sample, we suggest that atomic ordering may have occurred in parts of the sample over time, resulting in islands that consist of $\mathrm{Pb}_{\mathrm{x}} \mathrm{Se}_{\mathrm{y}} \mathrm{Te}_{\mathrm{z}}$ compounds with lattice constants and respective orientation relationships that are elastic mismatch energy minimizing. Just as in the III-V and II-VI QD systems we analyzed earlier [3-7] (and above), thermodynamics may possibly be the driving force behind structural transitions in ordinarily strained semiconductor QD predecessor structures [20].
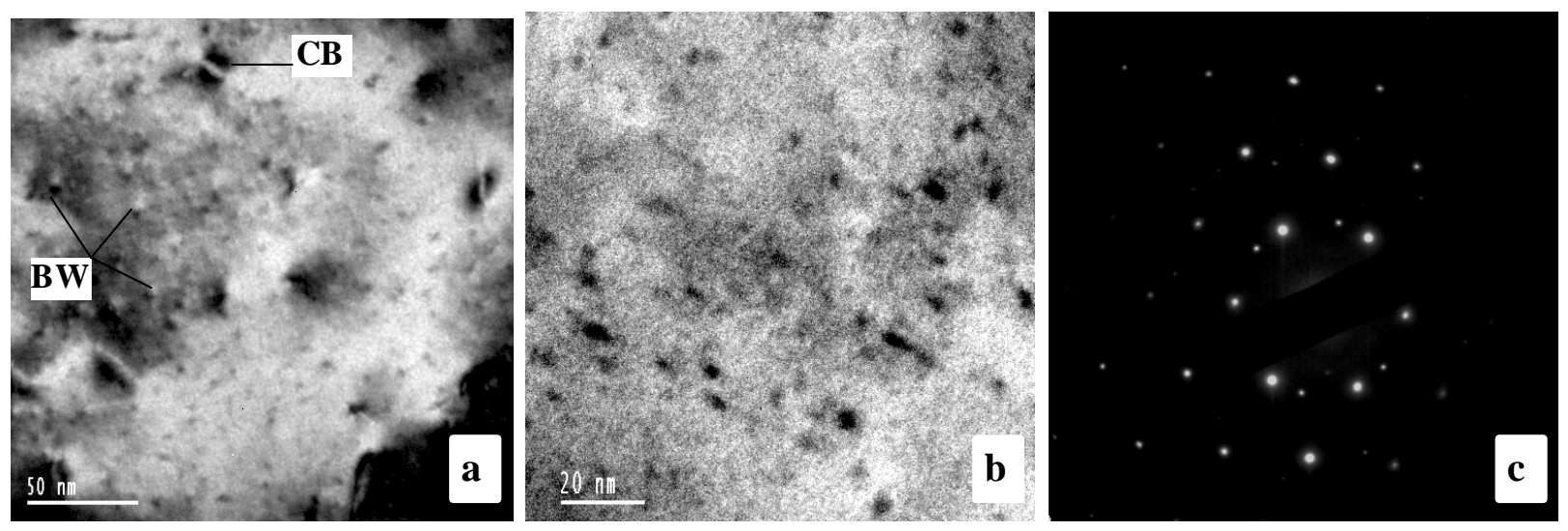

Figure 4. Near <111> plan view electron micrographs of the nominal PbSe islands on PbTe structure; (a) $\{220\}$ dark field diffraction contrast image of small ordinarily strained $\mathrm{Pb}(\mathrm{Se}, \mathrm{Te}) \mathrm{QD}$ predecessor islands, as revealed by typical "black-white" contrasts (marker BW and arrows), and of their somewhat larger (ordinarily strained) counterparts, which show the typical "coffee-bean" contrasts (marker CB and arrow); (b) $\{220\}$ dark field diffraction contrast image of small islands that do not show the typical strain field contrasts, indicating that these islands are not under (significant) lattice mismatch strains; (c) <111> zone axis SEAD pattern to (b) showing a variety of superlattice reflections that we assume to be due to $\mathrm{Pb}_{\mathrm{x}} \mathrm{Se}_{\mathrm{y}} \mathrm{Te}_{\mathrm{z}}$ compounds. The faint vertical lines in the diffraction pattern (c) are artifacts of the CCD camera used for the recording of this image.

\section{SUMMARY AND CONCLUSIONS}

The results of this study further contribute to the growing body of experimental observations in favor of atomic ordering and phase separation in epitaxial self-assembled compound semiconductor QDs and their predecessor island structures. Atomic ordering in epitaxial $(\mathrm{Cd}, \mathrm{Mn}, \mathrm{Zn})(\mathrm{S}, \mathrm{Se})$ with small or zero $\mathrm{Mn}$ content that manifests itself by luminescence peaks between 2 and $2.1 \mathrm{eV}$ may not be a rarely occurring phenomenon. Ordinarily strained $\mathrm{Pb}(\mathrm{Se}, \mathrm{Te})$ islands on $\mathrm{PbTe}$ were observed in diffraction contrast. Regions with small atomically ordered islands that consist probably of $\mathrm{Pb}_{\mathrm{x}} \mathrm{Se}_{\mathrm{y}} \mathrm{Te}_{\mathrm{z}}$ compounds were also identified in the same specimen. 


\section{ACKNOWLEDGMENTS}

Financial support from the Campus Research Board of the University of Illinois at Chicago (UIC) to PM and the National Science Foundation (Grant DMR-9733895) to NDB are gratefully acknowledged. Alan Nicholls (Electron

Microscopy Service of UIC) is thanked for experimental support. The authors are grateful to Malgorzata Dobrowolska and Jacek K. Furdyna (both University of Notre Dame) for the supply of the II-VI samples.

\section{REFERENCES}

1. A. Zunger and S. Mahajan, Atomic ordering and phase separation in epitaxial III-V alloys, in Handbook on Semiconductors (Elsevier Science B.V., 1994), Ed. T.S. Moss, Vol. 3, Volume Ed. S. Mahajan, p. 1447.

2. X. B. Zhang and S. K. Hark, J. Cryst. Growth 223, 512 (2001).

3. P. Möck, Y. Lei, T. Topuria, N. D. Browning, R. Ragan, K. S. Min, and H. A. Atwater, Physical Chemistry of Interfaces and Nanomaterials, Jin Z. Zhang, Zhong L. Wang, Eds., Proc. of SPIE, Vol. 4807, 71 (2002).

4. P. Möck, T. Topuria, N. D. Browning, R. J. Nicholas, and G. R. Booker, Mat. Res. Soc. Symp. Proc. 696, N8.8.1 (2002).

5. P. Möck, T. Topuria, N. D. Browning, M. Dobrowolska, S. Lee, J. K. Furdyna, G. R. Booker, N. J. Mason, and R. J. Nicholas, Appl. Phys. Lett. 79, 946 (2001).

6. P. Möck, T. Topuria, N. D. Browning, L. Titova, M. Dobrowolska, S. Lee, and J. K. Furdyna, J. Electron Mater. 30, 748 (2001).

7. P. Möck, T. Topuria, N. D. Browning, G. R. Booker, N. J. Mason, R. J. Nicholas, L. V. Titova, M. Dobrowolska, S. Lee, and J. K. Furdyna, Mater. Res. Soc. Symp. 642, J6.3.1 (2001).

8. K. Park, L. Salamanca-Riba, and B. T. Jonker, Appl. Phys. Lett. 61, 2302 (1992).

9. K. Cohen, S. Stolyarova, N. Amir, A. Chack, R. Beserman, R. Weil, and Y. Nemirovsky, J. Cryst. Growth 198/199, 1174 (1999).

10. H. S. Lee, J. Y. Lee, T. W. Kim, and H. L. Park, J. Cryst. Growth 233, 749 (2001).

11. M. S. Kwon and J. Y. Lee, J. Cryst. Growth 191, 51 (1998).

12. H. S. Lee, J. Y. Lee, T. W. Kim, D. U. Lee, D. C. Choo, and M. D. Kim, J. Appl. Phys. 91, 5657 (2002).

13. L. Salamanca-Young, D. L. Partin, and J. Heremans, J. Appl. Phys. 63, 1504 (1988).

14. T. Walter, A. G. Cullis, D. J. Norris, and M. Hopkinson, Phys. Rev. Lett. 86, 2381 (2001).

15. A. G. Cullis, D. J. Norris, T. Walter, M. A. Migliorato, and M. Hopkinson, Phys. Rev. B 66, 081305-1 (2002).

16. U. Håkanson, T. Sass, M. K-J. Johansson, M. E. Pistol, and L. Samuelson, Phys. Rev. B 66, 235308-1 (2002).

17. E. M. James and N. D. Browning, Ultramicroscopy 78, 125 (1999).

18. S-H. Wei and A. Zunger, Phys. Rev. B 39, 3279 (1989).

19. I. L. Krestnikov, M. Straßburg, M. Caesar, A. Hoffmann, U. W. Pohl, D. Bimberg, N. N. Ledentsov, P. S. Kop'ev, Zh. I. Alferov, D. Litvinov, A. Rosenauer, and D. Gerthsen, Phys. Rev. B 60, 8695 (1999).

20. The Lund University group [16] offers a different qualitative explanation for long range ordered microstructures in a III-V QD system. It is not clear if their samples were analyzed immediately after the growth. At least some of their samples were, however, baked at $120^{\circ} \mathrm{C}$ for 12 hours before the analyses. This might have converted growth induced short range order into the observed long range ordered microstructures.

21. C. S. Kim, M. Kim, J. K. Furdyna, M. Dobrowolska, S. Lee, H. Rho, L. M. Smith, H. E. Jackson, E. M. James, Y. Xin, and N. D. Browning, Phys. Rev. Lett. 85, 1124 (2000).

22. The fact that we observe neither (essentially) 2D remains of the nominally deposited 0.3 ML CdSe sheets nor 3D quantum dots with sphalerite structure in our HRTEM and Z-STEM investigations may be explained by averaging effects due to the [001] and $\langle 110\rangle$ projections in the former case and a small density in the latter case.

23. C. S. Kim, M. Kim, S. Lee, J. Kossut, J. K. Furdyna, and M. Dobrowolska, J. Cryst. Growth 214/215, 395 (2000).

24. G. Bacher, H. Schömig, M. K. Welsch, S. Zaitsev, V. D. Kulakovskii, A. Forchel, S. Lee, M. Dobrowolska, J. K. Furdyna, B. König, and W. Ossau, Appl. Phys. Lett. 79, 524 (2001).

25. L. V. Titova, J. K. Furdyna, M. Dobrowolska, S. Lee, T. Topuria, P. Moeck, and N. D. Browning, Appl. Phys. Lett. 80, 1237 (2002).

26. K. G. Chinyama, I. V. Bradley, K. P. O’Donnell, P. I. Kuznetsov, A. P. Chernushich, and V. Luzanov, J. Cryst. Growth 184/185, 298 (1998). 